\title{
Nutrigenomics: Moving towards Personalized Nutrition for Obesity Prevention
}

\author{
Anna DeBoer (University of Guelph) \\ News Reporter (HSI 2011-2012)
}

If you had the opportunity to simply provide a saliva sample, and in 6 weeks receive a list outlining your relative risk of developing type 2 diabetes and other diseases, would you take it? This opportunity is currently available from a US company called 23andme, which offers genetic testing to uncover disease risks using genetic markers with known associations. ${ }^{1}$ In response to public curiosity, commercial programs like 23andme and others apply genomic technologies, i.e. gene mapping and DNA sequencing, to disease prevention, but in truth they are only scratching the surface in the application of "omic" technologies to health. The term "omic" means "complete" or "all" based on a Greek suffix used to describe study areas in biology. ${ }^{2}$ Of the 50 or so "omic" terms coined so far, the field of nutrigenomics may soon be able to offer people more information on disease risk and prevention. ${ }^{2}$ Nutrigenomics allows researchers to study diet-gene interactions, or more generally, the role of genes in

\section{If you had the opportunity to simply provide} a saliva sample, and in 6 weeks receive a list outlining your relative risk of developing type

\section{2 diabetes and other diseases, would you}

\section{take it?}

response to changes in energy balance (food intake) or diet composition (micro- and macro-nutrients). ${ }^{3}$ What's more, with nutrigenomics it may one day be possible to make personalized dietary recommendations based on genotype in order to help people lose weight, optimize their health, or help prevent disease onset/exacerbation, especially for at risk populations prone to obesity and type 2 diabetes.

The field of nutrigenomics consists of two distinct but related areas called nutrigenetics and nutrigenomics. Nutrigenetics is the study of how an individual's genotype affects their response to diet, whereas nutrigenomics

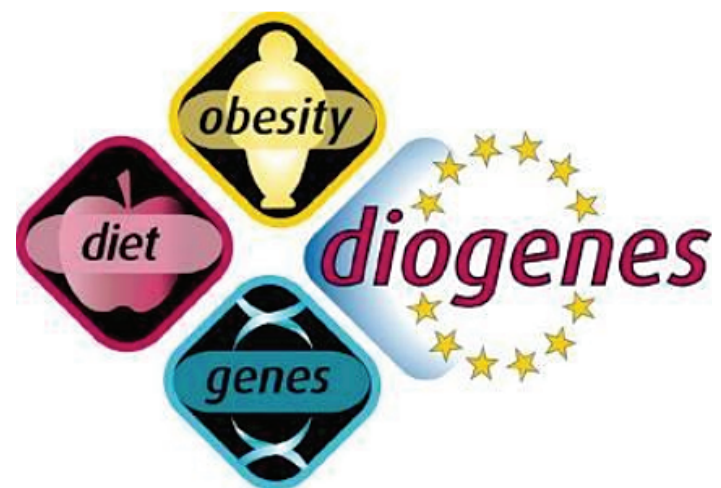

A recent large-scale European Framework VI clinical intervention study called Diet, Obesity and Genes identified unique gene expression profiles associated with maintainance of a new lower weight following a low-calorie dietary intervention.

is the study of how diet or specific nutrients affect gene expression, protein function, and subsequent downstream cell signalling pathways. ${ }^{2}$ Thus, it is now well accepted that to optimize health and prevent disease, one must consider environment and hereditary factors simultaneously. ${ }^{2}$ Importantly, nutrigenomic/nutrigenetic approaches allow researchers to study complex diseases like obesity and type 2 diabetes that are caused by a combination of genetic and environmental risk factors, such as diet.

It has been estimated that the heritability of obesity is between $40-70 \%{ }^{4}$ however, teasing out the cause of obesity is difficult because it is most commonly a polygenic (multi-factorial) disease with strong environmental factors. Polygenic forms of obesity are complex in nature where the adipose phenotype, a key tissue studied, is dependent on developmental, behavioural, environmental (e.g. diet composition), and genetic factors. ${ }^{5}$ In an effort to understand the causes of polygenic obesity, studies in the past few decades have unravelled genes that act as nutrient sensors, or genes that are implicated in obesity and diabetes pathogenesis. These findings have revealed that we have genes regulated by various micro- and macro-nutrients. ${ }^{6}$ For example, dietary fatty acids and their metabolites can bind directly to, and change the abundance/activity of specific transcription factors to control fat and carbohydrate metabolism. ${ }^{6}$ Moreover, nutrigenetic research in humans has revealed that in one's genotype there is genetic variation 
in nutrient-sensing pathways and obesity-related pathways that may alter their response to diet, and ultimately impact their risk of becoming obese or diabetic. ${ }^{7}$

Although it has been possible to study nutrient-sensing and obesity-related genes/pathways in isolation in the past, it has now become appreciated that every human genome consists of a unique mosaic of polymorphisms for these genes that need to be appreciated in combination to make dietary recommendations and assess risk. ${ }^{2}$ Dr. David Mutch, Assistant Professor of Nutrigenomics at the University of Guelph suggests that we need to get away from studying genes in an isolated "one at a time" manner, and rather study biological systems or networks. This is especially important in humans who are each genetically distinct (see Figure 1). Haplotypes consist of a unique combination of single nucleotide polymorphisms (SNPs), or different sites in the DNA sequence where individuals differ by one base pair that is inherited together in a specific chromosome region. ${ }^{8}$ Humans are about $99.9 \%$ genetically similar, and most genetic variation is accounted for by the 1 million SNPS found in the human genome, particularly by rare SNPs that have a frequency of around $1 \% .{ }^{8}$ Haplotypes can be used to look for genetic variation among humans, and hopefully identify genes or chromosome regions (alleles) that can account for some disease susceptibility. ${ }^{8}$ The International HapMap project is an international collaboration that has aimed to link genetic variation to disease states by creating a database of all known variation. ${ }^{8}$

Nutrigenomics researchers make use of the HapMap database to identify haplotypes associated with diet-gene interactions in obesity and type 2 diabetes. For example, Mutch and colleagues looked at polymorphisms in two human fatty acid desaturase genes (Fads1/2), which encode desaturase enzymes responsible for altering tissue lipid profiles. ${ }^{9}$ Increased delta 5 desaturase activity (Fads1) may lead to a greater production of lipids like arachadonic acid and its pro-inflammatory derivatives, whichare implicated in the pathogenesis of metabolic diseases including obesity and type 2 diabetes. ${ }^{9}$ Here, they looked at the relationship between SNPs for Fads $1 / 2$ and fatty acid desaturase activity in various ethnic populations from the Toronto Nutrigenomics and Health cohort. Most notably, they found a SNP in Fads1 where major/minor allele frequencies varied between Asians and Caucasians; however, regardless of ethnic background, carriers of the " $T$ " rather than the " $\mathrm{C}$ " allele were associated with greater desaturase activity. ${ }^{9}$ This study shows that there is genetic variation in the Fads gene locus in Caucasians and Asians, which is reflected by differences in desaturase activity. This research helps us understand why there are specific differences in people's lipid profiles, and may eventually contribute to improved dietary strategies to manage obesity characterized by altered lipid profiles. ${ }^{9}$

In addition to nutrigenetic research on haplotypes, human obesity is also being studied from a nutrigenomic standpoint (the effect of diet on genes). Mutch relates that associative nutrigenetic studies do not always agree probably because of differences in the population studied and the environmental factors affecting those populations. He goes on to propose that in order to further nutrigenomic research and reach the goal of personalized nutrition, largescale clinical trials are needed to detect rare genotypes

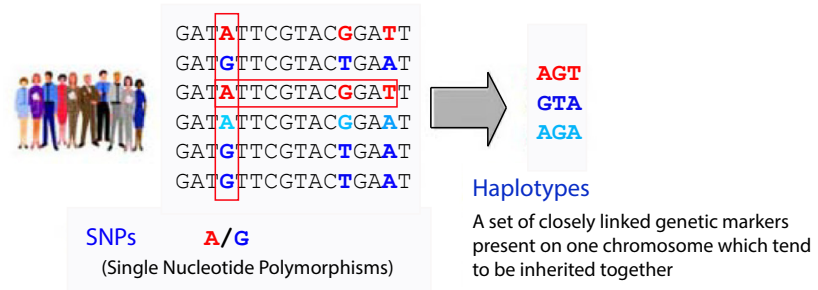

Figure 1: Each row of DNA sequence represents the chromosome of a different person. Coloured letters represent SNPs at specific chromosome locations. Image kindly made accessible through BioLicence open source information, http://omics.org/index. php/SNPenomics

and elucidate diet-gene interactions. A recent large-scale European Framework VI clinical intervention study called Diet, Obesity and Genes (abbreviated as DioGenes), recruited over 548 obese subjects to complete an 8-wk low-calorie diet, and then selected a subset of 40 nondiabetic women to identify changes in adipose tissue gene expression (mRNA; transcriptome) that took place during the diet. ${ }^{10}$ Moreover, the study went further to identify unique gene expression profiles in those who maintained their new weight following the low-calorie diet, versus those who re-gained the weight following the intervention. Using bioinformatic software to identify gene sets, a distinct profile of genes that are up or downregulated in response to certain stimuli (e.g. oxidative stress), Mutch and his colleagues were able to tease out candidate pathways that were modulated by the low-calorie diet in both weight maintainers and weight re-gainers (see Figure 2). ${ }^{10}$ In short, the authors were able to conclude that there may be a genetic basis for the ability to maintain weight loss following the low-calorie diet in women. ${ }^{10}$

In summary, current nutrition research in humans consists of cutting edge nutrigenetic studies like associative studies 


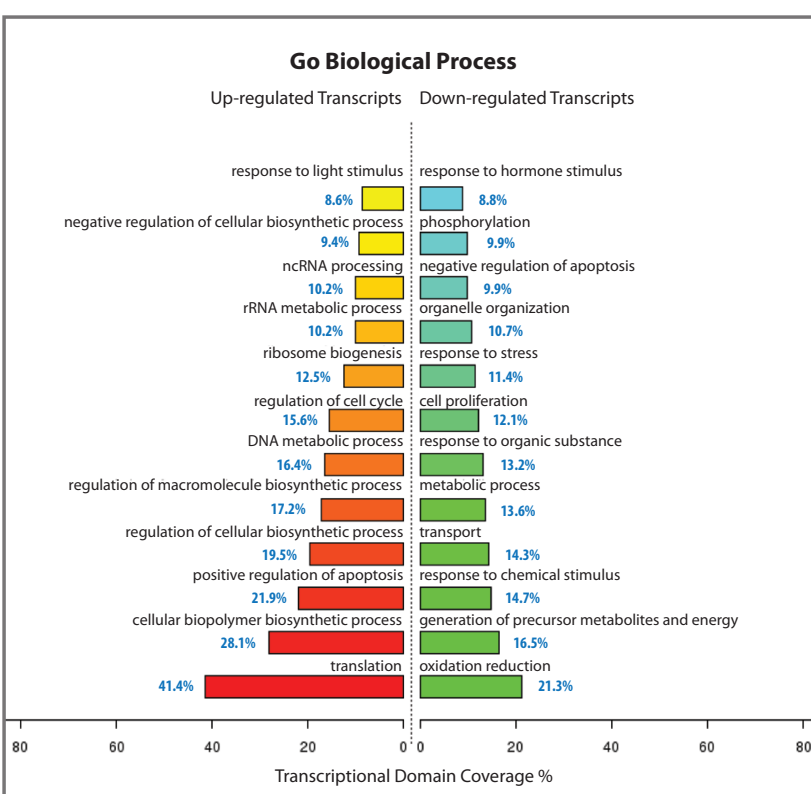

Figure 2: Bioinformatic output from Functional Network Analysis (๔ INSERM) showing the percent of up or down-regulated cellular pathways/processes from the Gene Ontology Biological Process database after weight loss in a treatment group of women compared to a control. This is done by analyzing microarray data of the transcriptome (unpublished data, image courtesy of Dr. D. Mutch).

that link haplotypes to disease states, and nutrigenomic studies like large clinical trials that aim to understand how dietary interventions affect gene expression and downstream signalling. These studies will unravel dietgene interactions and identify the relative contribution of genetics and environmental factors in complex diseases like obesity (polygenic) and type 2 diabetes. Finally, researchers like Mutch hope to see their recommendations for personalized nutrition based on nutrigenomic research come to fruition in the next decade.

\section{References}

1. How does 23andMe genotype my DNA? [internet]. c2002- 2011. (USA): 23andme Inc. [cited 2011 Dec 20]. Available from: https://www.23andme. com/you/faqwin/chip/

2. Mutch DM, Wahli W, Williamson G. 2005. Nutrigenomics and nutrigenetics: the emerging faces of nutrition. The Journal of the Federation of American Societies for Experimental Biology. 19:1602-1616.

3. Pérusse L, Bouchard C. Gene-diet interactions in obesity. 2000. American Journal of Clinical Nutrition. 72(5):1285S-1290S.

4. Allison DB, Faith MS, Nathan JS. 1996. Risch's lambda values for human obesity. International Journal of Obesity and Related Metabolic Disorders. 20:990-999.

5. Cheung WK. 2012. An Overview of Mongenic and Syndromic Obesities in Humans. Paediatric Blood Cancer. 58:122-128.

6. Mine $Y$, Miyashita K, Shahidi F, editors. 2009. Nutrigenomics and proteomics in health and disease. Ames (lowa): Wiley-Blackwell. 3-5p, 97p.

7. Mutch DM, Clément K. 2006. Unravelling the genetics of human obesity. PLoS Genetics [internet]. [cited 2011 Dec 20]; 2(12):e188. Available from: http://www.plosgenetics.org/article/info\%3Adoi\%2F10.1371\%2Fjournal. pgen.0020188

8. About HapMap [internet]. c2006-2011. National Center for Biotechnology Information, U.S. National Library of Medicine. Bethesda MD (USA): The International Hapmap Project [cited 2011 Dec 20]. Available from: http:// hapmap.ncbi.nlm.nih.gov/abouthapmap.html

9. Merino DM, Johnston H, Clarke S, Roke K, Nielsen D, Badawi A, El-Sohemy A, Ma DW, Mutch DM. 2011. Polymorphisms in FADS1 and FADS2 alter desaturase activity in young Caucasian and Asian adults. Molecular Genetics and Metabolism. 103(2):171-178.

10. Mutch DM, Pers TH, Ramzi Temanni M, Pelloux V, Marquez-Quinones A, Holst C, Alfredo Martinez J, et al. 2011. A distinct adipose tissue gene expression response to caloric restriction predicts 6 -mo weight maintenance in obese subject. American Journal of Clinical Nutrition. 94:1399-409.

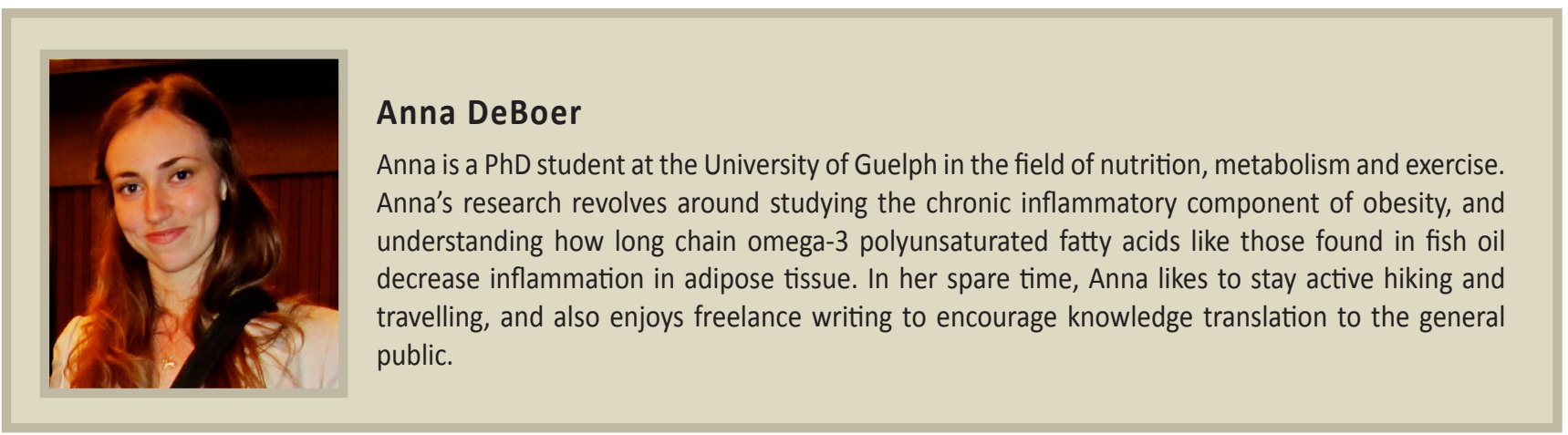

